\title{
Review of: "Pyrolysis of Lemon Peel Waste in a Fixed- bed Reactor and Characterization of Innovative Pyrolytic Products"
}

Chunxing Li

Potential competing interests: The author(s) declared that no potential competing interests exist.

The author applied pyrolysis technology for Lemon Peel Waste conversion to functional biochar and energy (syngas and oil) production. the characterization of pyrolysis products of biochar, syngas and oil are deeply studied, which is an interesting and meaningful work for LPW treatment. However, some minor errors should be revised before being accepted.

Line 49 a full stop is missing, checking the whole manuscript.

Line 77-78 this sentence should be re-write to make it correct and clear

Line 190 the title of results and discussion should be in a new row

Line 245-259 I suggest that this part should be discussed together with the following FTIR of the biochar samples, not presenting in a single part.

All the referrences should be carefully checked, especially for the name of the different journals (dot is missing in the journals)

The quality of the figures are low, it should be improved (unification】text...) 